\title{
Brief cognitive behavioural intervention delivered by nurses reduces overall symptoms in schizophrenia
}

Turkington D, Kingdon D, Turner T. Effectiveness of a brief cognitive-behavioural therapy intervention in the treatment of schizophrenia. British Journal of Psychiatry 2002 June; 180:523-7.

\section{QUESTION: Can community psychiatric nurses effectively deliver a brief cognitive behavioural therapy intervention for people with schizophrenia?}

Design

Randomised controlled trial.

\section{Setting}

6 psychiatric secondary care services in England, Ireland, Scotland and Wales.

\section{Participants}

422 people with schizophrenia in secondary care (defined using ICD-10 criteria). All were aged 18-65 years (mean 40 years); $23 \%$ women; $89 \%$ white. Exclusion criteria were deterioration; need for intensive home treatment; primary diagnosis of alcohol or drug dependence; organic brain disease or severe learning disability.

\section{Intervention}

Brief cognitive behavioural therapy or usual care. After 10 days of training, the intervention nurses visited patients for up to 6 hour-long sessions over 2-3 months. Carers received 3 optional sessions. All phases of cognitive behavioural therapy were included: assessment and engaging; developing explanations; case formulation; symptom management; adherence; core beliefs, and relapse prevention. Participants and their carers also received educational booklets.

\section{Main outcome measures}

Primary outcomes were overall symptoms (measured using Comprehensive Psychopathalogical Rating Scale), insight (Insight Rating Scale) and carer burden (Burden of Care Questionnaire). Secondary outcomes were schizophrenia symptoms (Schizophrenia Change Scale) and depression (Montgomery-Asberg Rating Scale).

\section{Main results}

The intervention group had improved overall symptoms, depression and insight (all $\mathrm{p}<0.05$; see table). There was no increase in suicide ideation and no difference between groups in symptoms of schizophrenia or burden of care.

\section{Conclusions}

Previous research suggests that interventions delivered by expert cognitive behavioural therapists are beneficial

Sources of funding: Pfizer Ltd.

For correspondence: D Turkington,

Department of

Psychiatry, University of

Newcastle-Upon-Tyne United Kingdom. douglas.turkington@ ncl.ac.uk for people with schizophrenia. This study found that

Number needed to treat with brief cognitive behavioural therapy to benefit one person with schizophrenia

\begin{tabular}{lc} 
& Number needed to treat \\
Improved overall symptoms & 13 \\
\hline Improved insight & 10 \\
\hline Improved depression scores & 9 \\
\hline
\end{tabular}

community psychiatric nurses can also deliver brief cognitive behavioural interventions safely and effectively.

\section{COMMENTARY}

There is evidence that nurses can competently deliver cognitive behavioural interventions for people with schizophrenia. ${ }^{1-2}$ Turkington et al found that overall symptoms improved after brief cognitive behavioural therapy delivered by Community Psychiatric Nurses, although contrary to previous studies, ${ }^{3}$ psychotic symptoms were not reduced.

Although the study was large and sufficiently powered, there are some caveats. First, the cost of the intervention is not mentioned. Nurses received 10 days of intensive training combined with weekly supervision. It would take many resources to make this intervention widely available. Second, longer term effects were not investigated. A longitudinal study is needed to assess whether brief cognitive behavioural therapy produces long lasting effects on symptom reduction, overall functioning, drug compliance, relapse and readmission rates. Third, there is no description of how nurses were selected. The qualifications, experience, motivation, commitment and abilities of nurses in the intervention group may have been different to those providing usual care.

It is important to account for patients' cultural values when integrating cognitive behavioural therapy into mental health services. In this study, the number of people from ethnic minorities was too small to allow meaningful comparison. As well as preparing educational material in different languages, it is important to question whether $\operatorname{cog}$ nitive behavioural therapy is acceptable to other cultures. Cultures may have different ways of expressing emotion. For example, Cheng suggests that Chinese people distrust 'talking therapy' and find it difficult to express emotions and engage in self-exploration. They may therefore find it difficult to participate in psychotherapy. ${ }^{4}$ Mental health professionals should be aware of these differences and deliver culturally sensitive care. Research is needed to assess patients' acceptance of and outcomes from cognitive behavioural therapy in various cultural groups.

Sally Wai-chi Chan, PhD MSc BSc RMN The Nethersole School of Nursing Chinese University of Hong Kong Shatin, Hong Kong

1 Hafner RJ, Crago A, Christensen D et al. Training case managers in cognitive-behaviour therapy. Australian and $N$ Zealand Journal of Mental Health Nursing

Sullivan J, Rogers P. Cognitive behavioural nursing therapy

D. O'Donnell A a al. Comparing the effectiveness of individual and group therapy in the treatment of depression. Adelaide: Joanna Briggs Institute for

4 Cheng LYC. Psychotherapy for the Chinese: Where are we going? In Cheng LY, Cheung F, Chen CN (eds). Psychotherapy or the Chinese. Hong Kong: Department of Psychiatry, Chinese University of Hong Kong, 1993: iv-viii 